Monte Carlo simulation of an optical coherence tomography signal in homogeneous turbid media

This content has been downloaded from IOPscience. Please scroll down to see the full text.

1999 Phys. Med. Biol. 442307

(http://iopscience.iop.org/0031-9155/44/9/316)

View the table of contents for this issue, or go to the journal homepage for more

Download details:

IP Address: 131.215.70.231

This content was downloaded on 20/07/2016 at 21:07

Please note that terms and conditions apply. 


\title{
Monte Carlo simulation of an optical coherence tomography signal in homogeneous turbid media
}

\author{
Gang Yao and Lihong V Wang $\dagger$ \\ Optical Imaging Laboratory, Biomedical Engineering Program Texas A\&M University, \\ College Station, TX 77843-3120, USA \\ E-mail: lwang@tamu.edu
}

Received 12 February 1999, in final form 8 July 1999

\begin{abstract}
The Monte Carlo technique with angle biasing is used to simulate the optical coherence tomography (OCT) signal from homogeneous turbid media. The OCT signal is divided into two categories: one is from a target imaging layer in the medium (Class I); the other is from the rest of the medium (Class II). These two classes of signal are very different in their spatial distributions, angular distributions and the numbers of experienced scattering events. Multiply scattered light contributes to the Class I signal as well as the Class II signal. The average number of scattering events increases linearly with the probing depth. The Class II signal decays much more slowly than the Class I signal whose decay constant is close to the total attenuation coefficient of the turbid medium. The effect of the optical properties of the medium on the Class I signal decay is studied.
\end{abstract}

\section{Introduction}

Optical low-coherence reflectometry is an imaging technique of high axial resolution (microns) and high dynamic range ( $>140 \mathrm{~dB}$ ) by use of a broadband light source and heterodyne detection (Sorin and Baney 1992). At the beginning of the 1990s, optical low-coherence reflectometry was further extended to acquire two-dimensional tomographic images in biological tissue, a method commonly called optical coherence tomography (OCT) (Huang et al 1991). Since then OCT has been successfully applied in ophthalmology for retinal imaging (Puliafito et al 1995). In recent years, its application in highly scattering biological tissue such as skin tissue has attracted much attention. However, such applications are limited by the penetration depth, unlike its successful applications in transparent ocular organs. It is believed that multiple scattering, which becomes dominant at large depths, is the fundamental limitation preventing OCT from reaching a large probing depth in turbid media (Yadlowsky et al 1995).

In order to understand the governing physical process and to better interpret the OCT signal in highly scattering media, some theoretical models have been developed. Pan et al (1995) established the relationship between the path-length resolved reflectance signal and the OCT signal using linear system theory. They used a Monte Carlo technique to simulate the path-length resolved reflectance but did not separate the effects of the singly scattered light and the multiply scattered light. Schmitt and Knuttel (1997) described an OCT model based on Huygens-Fresnel diffraction optics. They split the OCT signal into the summation of singly back-scattered light (coherent) and multiply scattered light (partially coherent). The effect of

$\dagger$ Author to whom correspondence should be addressed. 
multiple scattering on the formation of speckle patterns and the degradation of image contrast were demonstrated.

In reality, light scattering in turbid media is a complex process, and it is only an approximation to assume that the OCT signal is from single back-scattering alone. Photons still contribute to the OCT signal after a limited number of scattering events. In a recent paper, Smithies et al (1998) used Monte Carlo simulation to model the OCT signal in homogeneous turbid media. The multiple scattering effects are clearly demonstrated in terms of the spreading of the point spread function (PSF).

In this paper, we directly simulate the contribution of the multiple-scattered light to the OCT signal. The OCT signal is divided into two parts: one includes the light coming from the target layer in the medium, and the other includes the light coming from the background other than the target layer. The Monte Carlo technique with angle biasing (Hendricks and Carter 1985 ) is applied to speed up the simulation and reduce the statistical variance.

\section{Method}

\subsection{OCT model}

An OCT system is basically a Michelson interferometer with a low-coherence light source (figure 1). The light from the reference arm and the sampling arm can interfere at the detector only when the difference of their optical path-lengths is within the coherence length of the light source. Therefore, the axial resolution of OCT is determined by the coherence length of the light source.

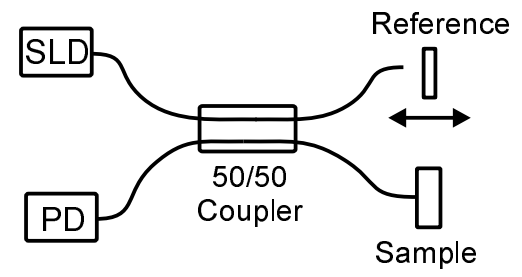

Figure 1. Schematic diagram of an OCT system. SLD: super-luminescence diode; PD: photodiode.

In the simulation, it is assumed that the probing fibre is in direct contact with the turbid medium, which is similar to the OCT set-up in a previous experiment (Schmitt et al 1993). The radius of the probing fibre is $10 \mu \mathrm{m}$. For simplicity, the fibre is assumed to emit a pencil beam. The light back-scattered from the sample is divided into two parts: Class I and Class II (figure 2).

The Class I photons refer to the photons scattered from a specific target layer whose central depth corresponds to the path-length of the reference arm and whose thickness is determined by

$$
2 n \Delta z=l_{c}
$$

where $n$ is the refractive index of the medium, $l_{c}$ is the coherence length of the light source in a vacuum and $\Delta z$ is the thickness of the layer. The Class I light is a useful signal in OCT because it is scattered from the target layer and contains the local optical information. It is necessary to point out that the Class I photons are from the target layer, but they cannot be from anywhere in this layer because their optical path-length must be within the range $\left[p-l_{c} / 2\right.$, $\left.p+l_{c} / 2\right]$, where $p$ is the path-length of the reference arm. 


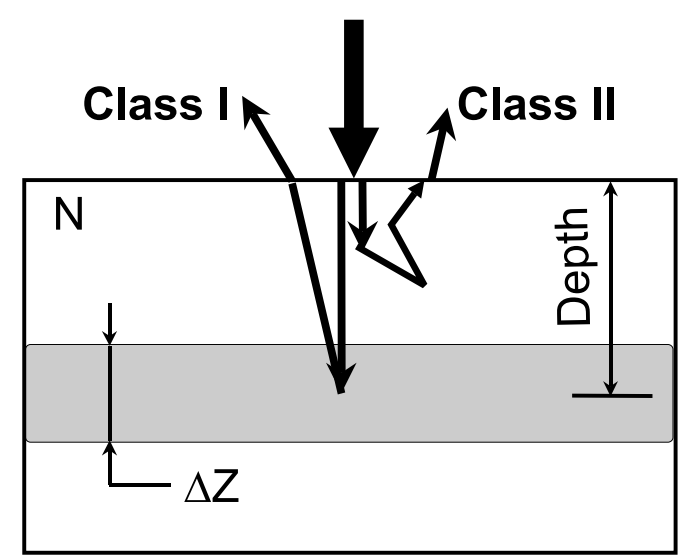

Figure 2. Composition of the OCT signal, where I represents the light from the specified target layer (Class I), and II represents the light from the rest of the medium (Class II).

The Class II photons are the photons scattered from the rest of the medium above the target layer and its optical path-length is also within the range $\left[p-l_{c} / 2, p+l_{c} / 2\right]$. Because the path-length difference between this part of the light and the reference light is within the coherence length, the Class II light can also be detected. The Class II light contributes to the noise in OCT signal because it is not scattered from the target layer and does not furnish any local information about the target layer. This part of the light is responsible for the degradation of the contrast of OCT images and may overwhelm the Class I signal at large probing depths.

The OCT signal can be written as (Pan et al 1995)

$$
I_{d}(\tau)=I_{s}+I_{r}+2\left(I_{s} I_{r}\right)^{1 / 2} \operatorname{Re}\left[V_{\mathrm{mc}}(\tau)\right]
$$

where $\tau$ is the time delay between the reference arm and the sampling arm, $I_{s}$ and $I_{r}$ are the ensemble averaged light intensities from the reference arm and the sampling arm respectively and $V_{\mathrm{mc}}$ is the mutual coherence function of the light from the two arms and is assumed to be rectangular for simplicity. Equation (2) indicates that the OCT signal is proportional to the square root of the diffuse reflectance $I_{s}$. In our model, $I_{s}$ is the summation of the Class I light $\left(I_{1}\right)$ and the Class II light $\left(I_{2}\right)$ :

$$
I_{s}=I_{1}+I_{2} \text {. }
$$

The light whose path-length difference with the reference path-length is beyond the coherence length is simply discarded because it does not contribute to the OCT signal.

In our OCT model, two factors affect the detection solid angle of the receiver. One is the coupling angle of the single-mode fibre; the other is the antenna theorem of heterodyne detection. In our case, the antenna theorem can be written as (Siegman 1966)

$$
(\Delta \theta)^{2} \approx \lambda^{2} / A
$$

where $A$ is the detector area, $\Delta \theta$ is the acceptance angle of the detector and $\lambda$ is the wavelength. In this simulation, the detector radius is $10 \mu \mathrm{m}, \lambda=1.5 \mu \mathrm{m}$, then $\Delta \theta \approx 5^{\circ}$ is chosen as the detection angle of the receiver.

\subsection{Angle biased Monte Carlo simulation}

Monte Carlo simulation has been proved to be an accurate method to study photon-tissue interaction. Because biological tissues usually have very large anisotropy factors, light 

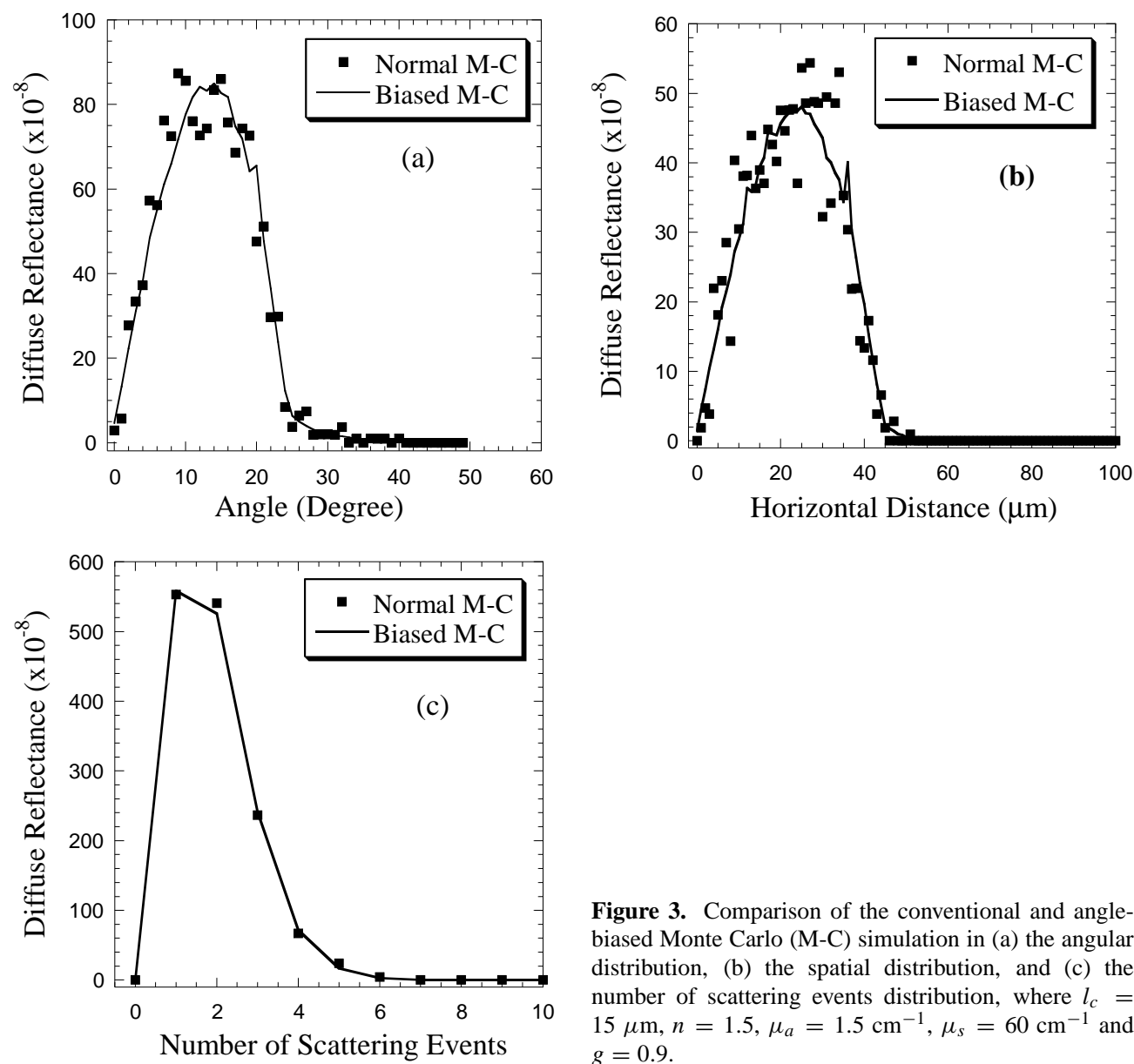

Figure 3. Comparison of the conventional and anglebiased Monte Carlo (M-C) simulation in (a) the angular distribution, (b) the spatial distribution, and (c) the number of scattering events distribution, where $l_{c}=$ $15 \mu \mathrm{m}, n=1.5, \mu_{a}=1.5 \mathrm{~cm}^{-1}, \mu_{s}=60 \mathrm{~cm}^{-1}$ and $g=0.9$.

undergoes highly forward scattering and has a small chance of being back-scattered. In addition, OCT modelling requires very high optical path length resolution (of the order of the coherence length). Therefore, the photon yield is extremely low in OCT modelling. In order to accelerate the computation, we applied a variance reduction technique called 'angle biased' sampling (Hendricks and Carter 1985). The basic idea is to use an artificial scattering phase function to replace the true phase function when sampling the scattering angle and then update the photon weight according to

$$
w^{*}=\frac{f(\theta, \varphi)}{f^{*}(\theta, \varphi)} w
$$

where $\theta(0 \leqslant \theta \leqslant \pi)$ and $\theta(0 \leqslant \theta \leqslant \pi)$ are the photon deflection angle and azimuthal angle of a single scattering event, $f(\theta, \varphi)$ is the true phase function for the photon scattering, $f^{*}(\theta, \varphi)$ is the artificial phase function used in the sampling, $w$ is the photon weight corresponding to the phase function $f(\theta, \varphi)$, and $w^{*}$ is the photon weight corresponding to the phase function $f^{*}(\theta, \varphi)$.

In our simulation, the Henyey-Greenstein phase function (Wang et al 1995) is chosen to sample the photon scattering angle

$$
p(\cos \theta)=\frac{1-g^{2}}{2\left(1+g^{2}-2 g \cos \theta\right)^{3 / 2}}
$$


where $g$ is the anisotropy factor and $\theta$ is the photon deflection angle of a single scattering event. $p(-\cos \theta)$ is applied as the artificial phase function. In other words, after $\cos \theta$ is sampled with $p(\cos \theta),(-\cos \theta)$ is actually used to calculate the direction of travel of the photon packet. Meanwhile, the photon weight is adjusted by

$$
w^{*}=\left(\frac{1+g^{2}+2 g \cos \theta}{1+g^{2}-2 g \cos \theta}\right)^{3 / 2} w .
$$

The same technique was used in the simulation of confocal microscopy (Schmitt and BenLetaief 1996). Our simulation results show that this method has greatly improved the statistical properties of the calculated diffuse reflectance and significantly reduced the computation time.

\subsection{Simulation process}

The specular reflection from the fibre-tissue interface is neglected in our simulation. The basic Monte Carlo simulation method has been described in detail elsewhere (Wang et al 1995). In the modelling process, a layer in the medium is specified with a central depth corresponding to a predefined optical path-length in the reference arm (figure 2). After a photon package is launched, it is traced by the conventional Monte Carlo method. Whenever a photon hits the target layer, it is labelled and the angle-biased sampling technique is applied to sample its scattering angle. Otherwise, the photon scattering is sampled by the normal Henyey-Greenstein phase function. The optical path-length of each photon packet is also traced. The photon is discarded whenever the difference between its path-length and the reference path-length is beyond the source coherence length. The labelled photons reaching the detector are scored into the Class I signal and the unlabelled photons are scored into the Class II signal. The signal intensity is calculated as the square root of the diffuse reflectance, and the decay constant of the signal is calculated as the derivative of the signal intensity with respect to the round-trip probing depth. When the photons leave the surface of the turbid medium, the horizontal distance from the point of light incidence and the exit angle with respect to the normal to the surface of the medium are recorded for calculation of the spatial or angular distribution of the signal.

Unless otherwise indicated, the optical parameters used in the simulation are: the coherence length of the light source in vacuum $l_{c}=15 \mu \mathrm{m}$; the refractive index of the turbid medium $n=1.5$, the absorption coefficient $\mu_{a}=1.5 \mathrm{~cm}^{-1}$, the scattering coefficient $\mu_{s}=60 \mathrm{~cm}^{-1}$, the total attenuation coefficient $\mu_{t}=\mu_{a}+\mu_{s}=61.5 \mathrm{~cm}^{-1}$, and the anisotropy factor $g=0.9$.

\section{Results}

\subsection{Verification of the simulation method}

The spatial and angular distributions of the Class I signal for small probing depths are calculated and compared with the results of conventional Monte Carlo simulation. The comparison for a large probing depth is difficult because the statistical error in the conventional Monte Carlo method is too large. Figure 3 shows the results for a depth of $100 \mu \mathrm{m}$. A total of $10^{8}$ incident photons are sampled in the simulation. Obviously, the angle-biased sampling technique yields much smoother results than the conventional approach. The calculated total reflectances by the two methods are in agreement with each other. For example, for the depth of $100 \mu \mathrm{m}$, the total reflectances are $1.425 \times 10^{-5} \pm 0.037 \times 10^{-5}$ and $1.417 \times 10^{-5} \pm 0.010 \times 10^{-5}$ respectively. 


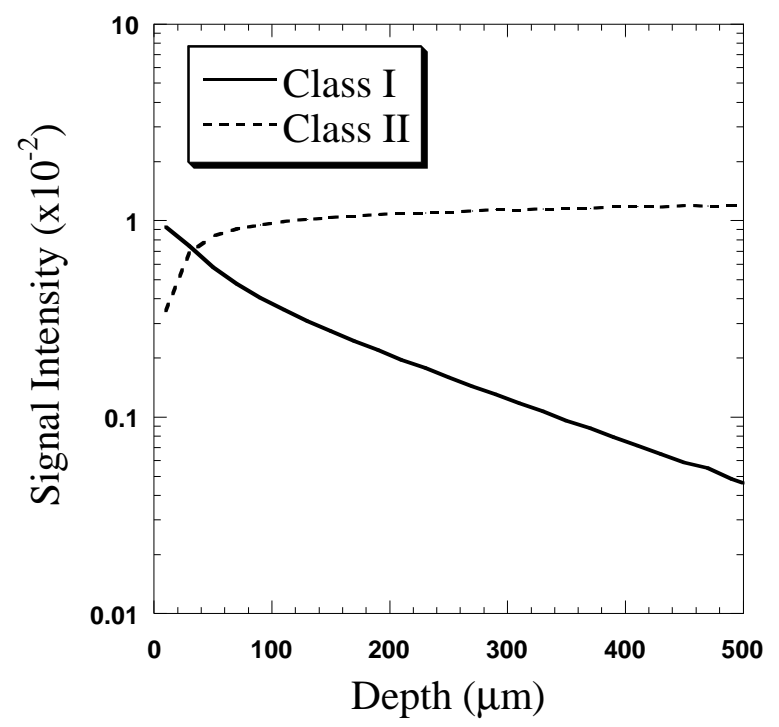

Figure 4. Spatially and angularly integrated Class I and Class II signals versus the probing depth. The simulation parameters are the same as those in figure 3.

\subsection{OCT signal profiles for different probing depths}

Figure 4 shows the spatially and angularly integrated back-scattering signals. It can be seen that the integrated Class I signal intensity decreases almost exponentially beyond $\sim 100 \mu \mathrm{m}$ in depth, but the integrated Class II signal increases as the depth increases. The integrated Class II signal begins to exceed the integrated Class I signal at a very small probing depth. Fortunately, these two classes of photons have very different spatial and angular distributions, which makes OCT possible.

The spatial distributions of Class I and Class II signals are shown in figure 5. It is clear that the Class II signal has a much broader spatial distribution than the Class I signal. Although the spatial distributions of both signals become broadened as the probing depth increases, the Class II signal is broadened much faster than the Class I signal. Obviously, we can limit the detection area to reject most of the Class II signal. In our simulation, the detector is a single-mode fibre which has a radius of $10 \mu \mathrm{m}$. In other words, if the spatial distance of an output photon from the incident point is greater than $10 \mu \mathrm{m}$, it will not be detected. With this parameter, the normalized angular distributions of the Class I and Class II signals with angular resolution of $5^{\circ}$ are shown in figure 6 . Clearly, the Class II signal has a much wider angular distribution than the Class I signal. Although the absolute intensity decreases as the probing depth increases, the distribution profiles are almost the same. Based on this result, the Class II photons can be further rejected by limiting the acceptance angle of the detector. Actually this is a natural restriction in the case of a single mode fibre which has a limited coupling angle. For an optimal detection angle, the detector should accept as much of the Class I light as possible and reject as much of the Class II light as possible. There is a trade off because when the detection angle is narrowed to reject the Class II photon, the received Class I signal is reduced, and vice versa.

If the detection area is limited to a radius $r_{d}=10 \mu \mathrm{m}$, and the detection angle is limited to $\theta_{d}=5^{\circ}$, then the Class II signal intensity exceeds the Class I signal intensity at the depth of approximately $500 \mu \mathrm{m}$ (figure 7). This critical depth, at which the Class II light intensity is 

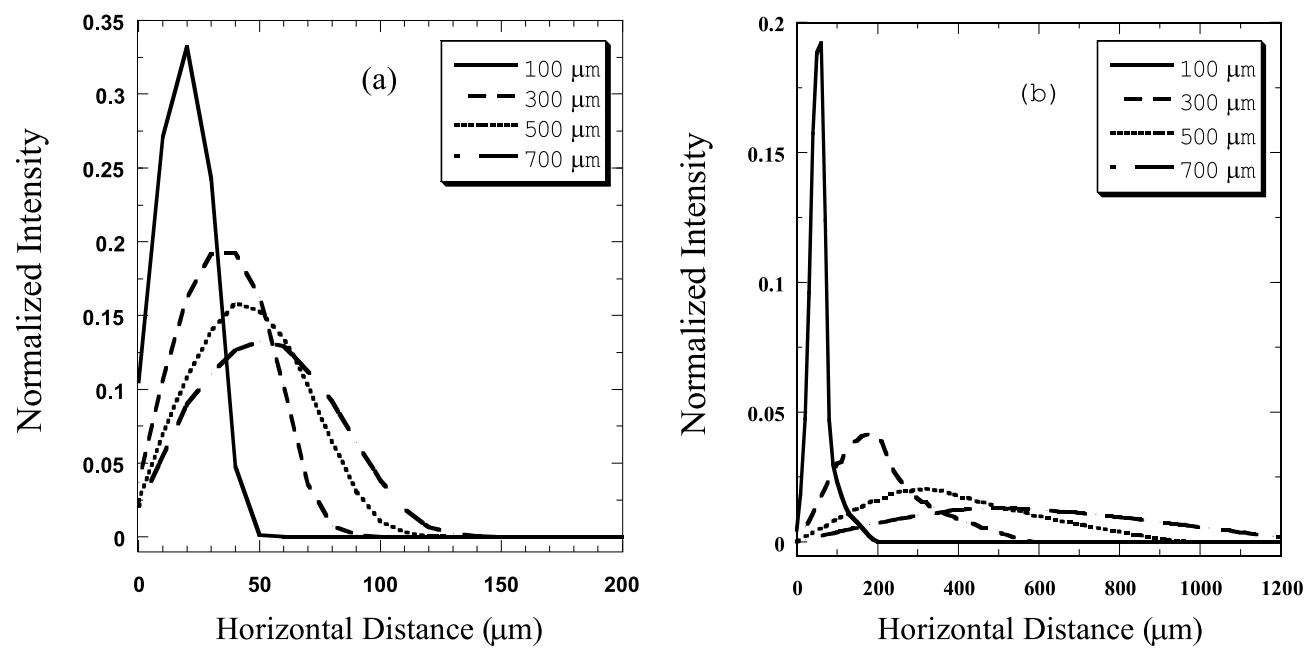

Figure 5. Normalized spatial distributions of (a) the Class I and (b) the Class II signals for various probing depths. The simulation parameters are the same as those in figure 3 .
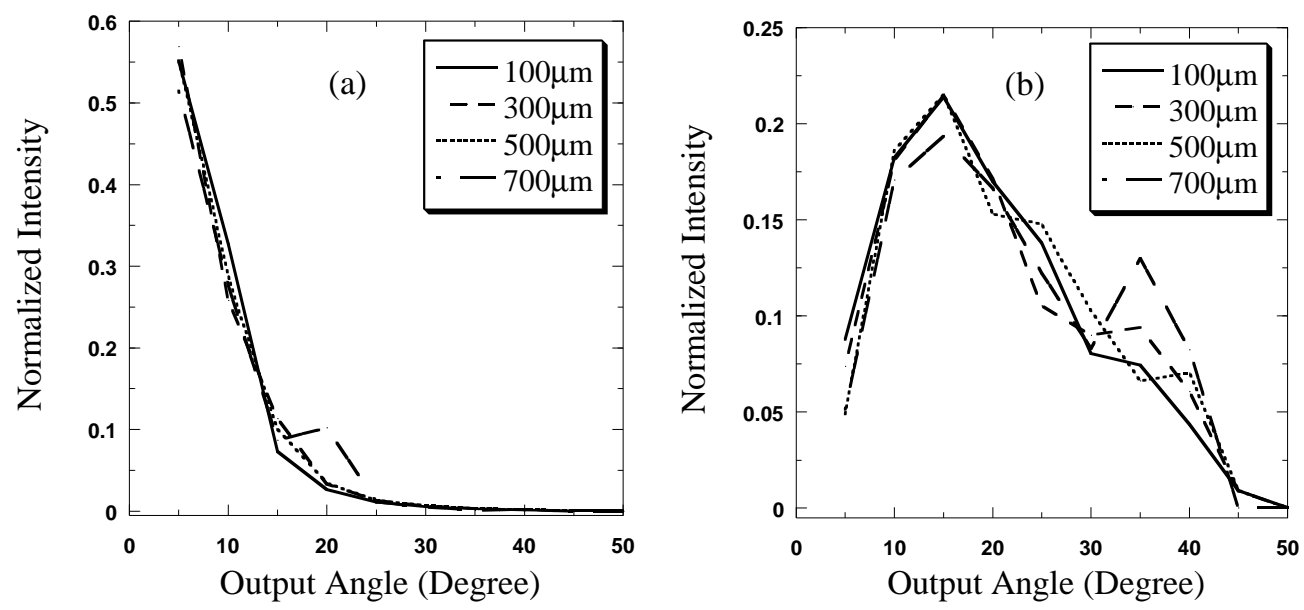

Figure 6. Normalized angular distributions of (a) the Class I signal and (b) the Class II signal for various probing depths, where $r_{d}=10 \mu \mathrm{m}$ and the other simulation parameters are the same as those in figure 3 .

equal to the Class I light intensity, should actually be larger because the numbers of scattering events relating to the Class II and the Class I signals are different. The Class II photons undergo more scattering events than the Class I photons. The average number of scattering events of the Class II signal increases faster with the probing depth than that of the Class I signal (figure 8). Because multiple scattering depolarizes the light, the OCT signal which is actually measured is less than the calculated signal intensity. In an idealized scenario where we assume that the polarization of the Class II light is completely randomized for large probing depths while the polarization of the Class I light is maintained, the critical depth becomes approximately $700 \mu \mathrm{m}$ (figure 7), at which the image contrast decreases to 50\%. When the light in both the classes is partially polarized, the critical depth is between the two extremes at 500 and $700 \mu \mathrm{m}$. 


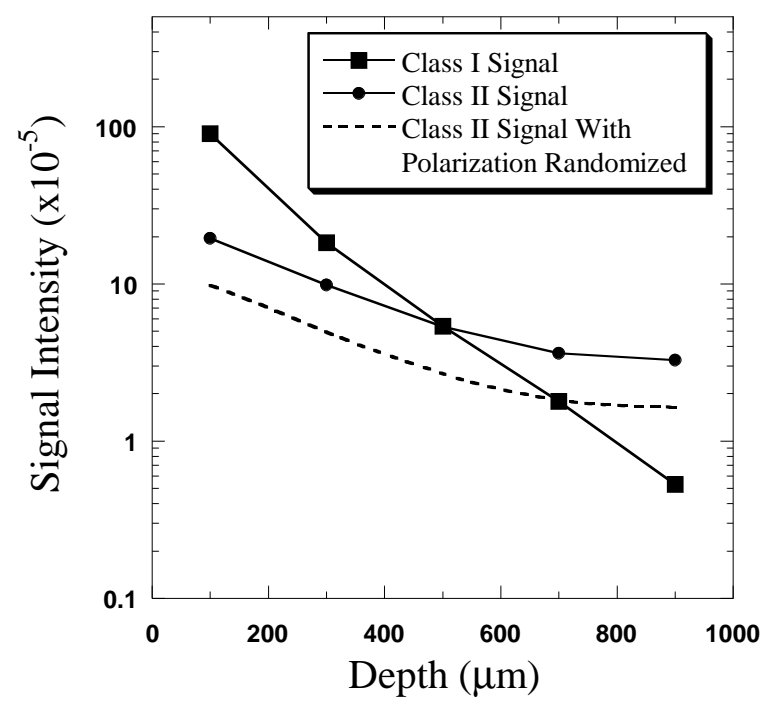

Figure 7. Class I and Class II signals versus the probing depth, where $\theta_{d}=5^{\circ}$ and the other simulation parameters are the same as those in figure 6.

Figure 8 indicates that the Class I light also contains multiply scattered photons. The average number of scattering events in the Class I signal returned from a depth of $200 \mu \mathrm{m}$ is two, and this increases linearly with the probing depth. Owing to the requirement of matching the optical path-lengths, these multiple scattering events in the Class I light must be smallangle scattering. Otherwise, their optical path-length difference with the reference arm would exceed the coherence length and would not contribute to the OCT signal. Therefore, these multiply scattered Class I photons will still largely preserve their polarization properties and hence cannot be rejected by the detection system. Theoretically, only the singly back-scattered photons can directly furnish the exact local optical information because they do not experience interactions elsewhere. Conversely, the multiply scattered photons do not furnish accurate local optical information because they experience interactions at multiple sites.

Figure 9 shows the decay of the Class I signal with different numbers of scattering events. The singly back-scattered light is the strongest for small probing depths but is soon exceeded by multiply scattered light as the probing depth increases. Obviously, the probability of multiple scattering increases with imaging depth. It can be seen that all the curves decay almost exponentially. However, the decay constant of the singly back-scattered light is much greater than the total attenuation coefficient $\mu_{t}$. In this example, the decay constant is $\sim 90 \mathrm{~cm}^{-1}$, while $\mu_{t}=61.5 \mathrm{~cm}^{-1}$. This phenomenon can be easily understood by considering the geometric scheme of the OCT system in this simulation.

In general, the detected signal intensity depends on three factors: (a) the number of photons hitting the target layer; (b) the portion of these photons that are back-scattered; and (c) the portion of these back-scattered photons that reach the detection area. For the OCT scheme used in this simulation, we can assume that the singly back-scattered photons from different depths have the same angular distribution because they have the same scattering probability distribution. The singly back-scattered light from a large depth has a large output area on the surface and hence less is captured by the detector. This effect leads to a decay constant of the singly back-scattered light greater than $\mu_{t}$. For the multiply scattered component of the Class I signal, the collective effect of these factors leads to a decay constant that is slightly different from $\mu_{t}$. 


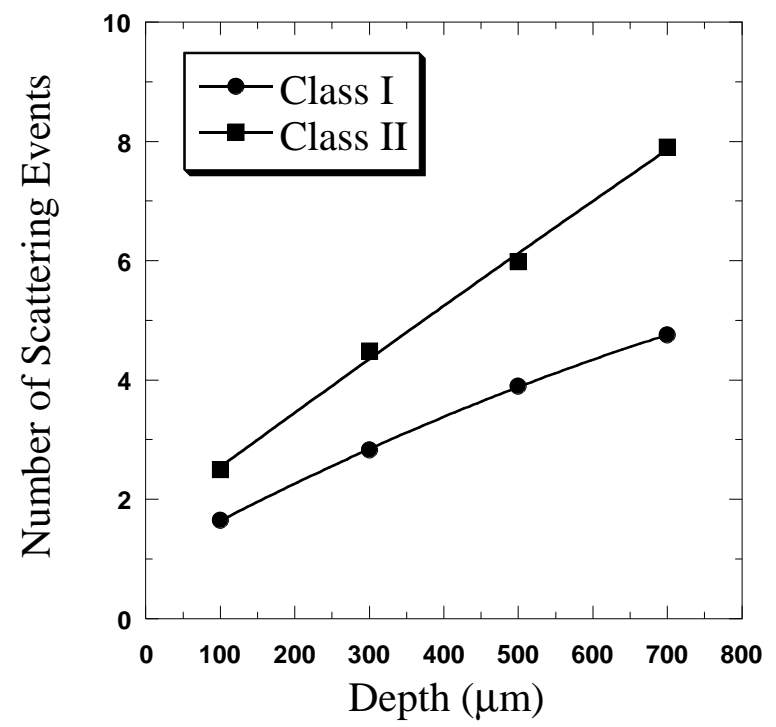

Figure 8. Weighted average numbers of scattering events of Class I and Class II signals versus the probing depth. The simulation parameters are the same as those in figure 7.

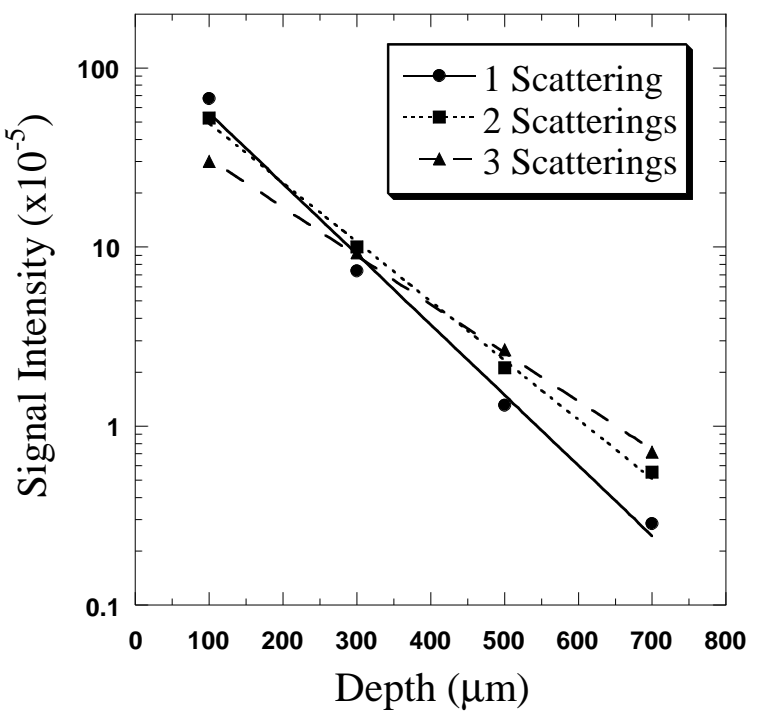

Figure 9. Decay of the Class I signal intensity with different numbers of scattering events versus the probing depth. The simulation parameters are the same as those in figure 7.

\subsection{Decay of the Class I signal with the probing depth}

Because Class I light can furnish information about local optical properties, it should be useful to study its variation with the probing depth. Figure 10 shows one of the typical simulation results. The signal decay is close to exponential with increase in probing depth. At large depths, the simulation results have very large variances because few photons are recorded. The calculated decay constant (the slope of the curve) is close but not exactly equal to the total 


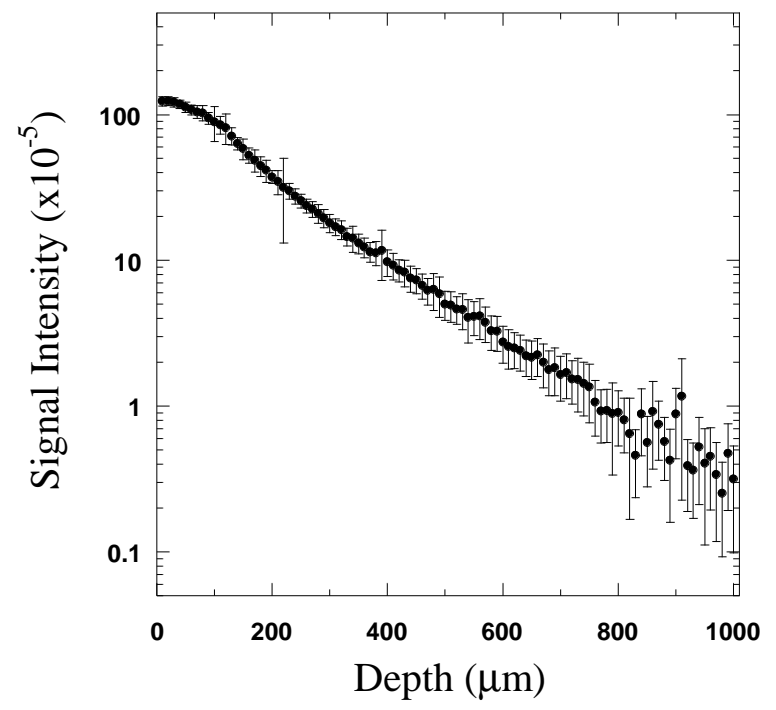

Figure 10. Class I signal intensity versus the probing depth. The simulation parameters are the same as those in figure 7.

attenuation coefficient $\mu_{t}$ and also has some very slight variations with the probing depth. The decay is a little slower at large depths because the multiply scattered light becomes dominant as stated in the previous section. Generally speaking, the decay constant of the Class I signal depends on the specific OCT system configuration as well as the optical properties of the turbid medium.

\subsection{Effects of coherence length and optical properties on the attenuation of the Class I signal}

The coherence length of the light source determines the axial resolution of an OCT system. In order to improve the axial resolution, a light source with a small coherence length is needed. It is easy to understand that the OCT signal intensity is smaller for a smaller coherence length because there is less light contributing to the signal at a specific reference path-length. In addition, the simulation result in figure 11(a) shows that the decay constant of the signal is also slightly greater for a smaller coherence length. In other words, the Class I signal decays faster for a smaller coherence length.

Figure 11(b) shows the decay of the Class I signal for three different values of the absorption coefficient $\mu_{a}=0.15 \mathrm{~cm}^{-1}, 1.5 \mathrm{~cm}^{-1}$ and $15 \mathrm{~cm}^{-1}$ while the scattering coefficient is kept constant $\left(\mu_{s}=60 \mathrm{~cm}^{-1}\right)$ in the calculation. When $\mu_{a}$ is small compared with $\mu_{s}$, the decay curves are insensitive to $\mu_{a}$. When $\mu_{a}$ is large, the signal intensity is weaker and decays faster as $\mu_{a}$ increases. The decay constants are calculated to be $60.8 \mathrm{~cm}^{-1}, 63.0 \mathrm{~cm}^{-1}$ and $77.2 \mathrm{~cm}^{-1}$, respectively. This result indicates that the decay constant of the Class I signal is correlated more with $\mu_{t}$ than with $\mu_{s}$.

Figure 11(c) shows the decay of the Class I signal for three values of the anisotropy factor: $g=0.7,0.8$ and 0.9 . The simulation results indicate that the decay constant of the Class I signal is also related to the anisotropy factor. The signal decays faster for a smaller $g$. As stated in section 3.2, the signal intensity depends on three factors. Calculation shows that the number of photons hitting a target layer changes very little for different $g$ values. However, the back-scattering probability increases as the anisotropy factor decreases. Therefore the Class I 

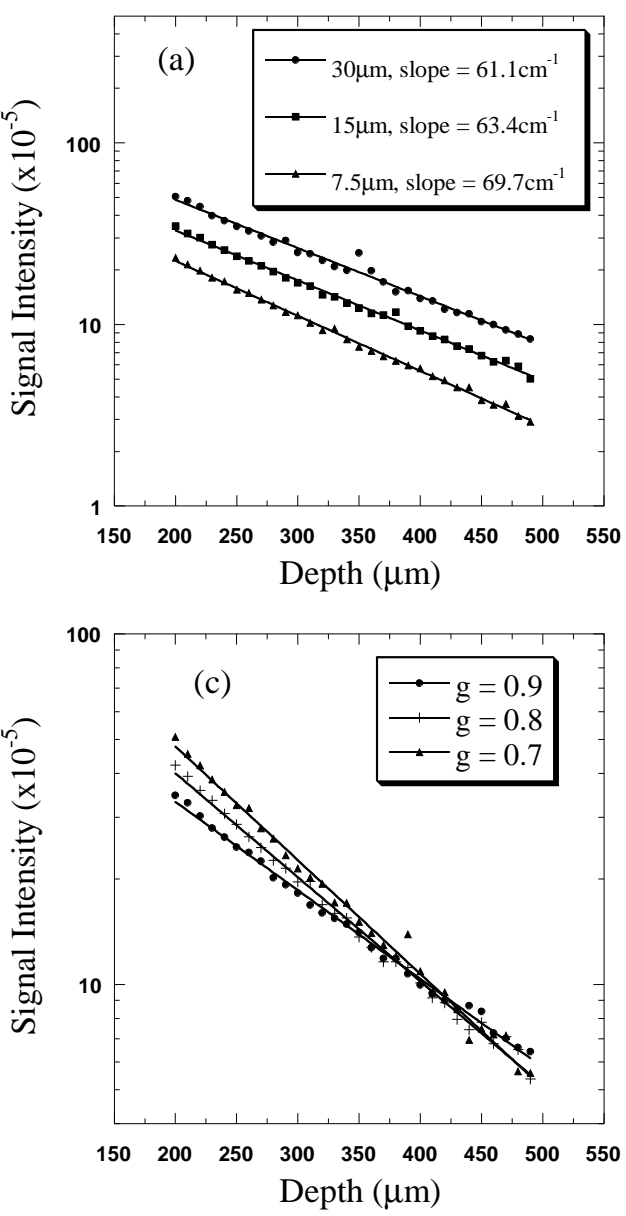

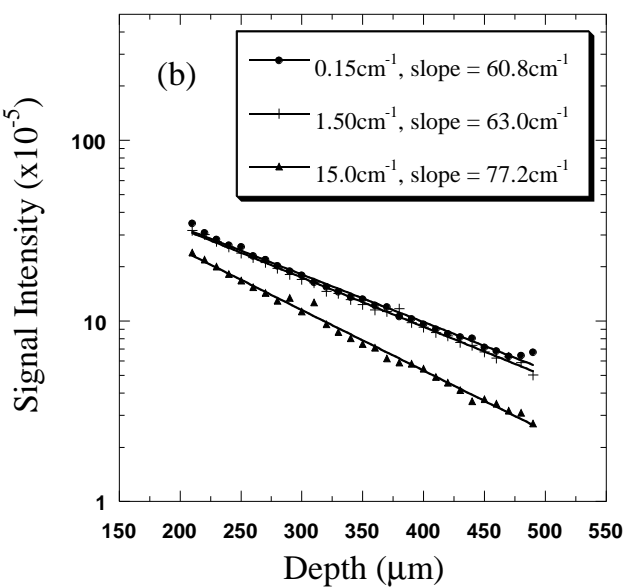

Figure 11. Class I signal intensity versus the probing depth for varying (a) coherence length $l_{c}$, where $\mu_{a}=$ $1.5 \mathrm{~cm}^{-1}, \mu_{s}=60 \mathrm{~cm}^{-1}$ and $g=0.9$; (b) absorption coefficient $\mu_{a}$, where $l_{c}=15 \mu \mathrm{m}, \mu_{s}=60 \mathrm{~cm}^{-1}$ and $g=0.9$; (c) anisotropy factor $g$, where $l_{c}=15 \mu \mathrm{m}$, $\mu_{a}=1.5 \mathrm{~cm}^{-1}$ and $\mu_{s}=50 \mathrm{~cm}^{-1}$. The parameters that are shared by the three sets of simulations include: $n=1.5, r_{d}=10 \mu \mathrm{m}$ and $\theta_{d}=5^{\circ}$.

signal should be larger for a smaller anisotropy factor. Although this is true for small probing depths, the situation is different for a sufficiently large probing depth because of the large difference in the angular distributions of the photons hitting the layer. For a small $g$ factor, the photons have a large probability of deviating far from their original directions and hence have a small probability of hitting the detector after being back-scattered. As shown in figure 12, the angular distributions have significant differences at small angles, which corresponds to the being light scattered several times because the light with no scattering is still collimated $\left(0^{\circ}\right.$ angle $)$ and the light with many scattering events has a broad angular distribution. There are far fewer photons hitting the target layer with small incident angles for a smaller $g$. As indicated in section 3.2, the photons experiencing several scattering events become dominant for large probing depths. Therefore, the loss of such photons for a smaller anisotropy factor leads to a faster decay of the Class I signal.

Figure 13 shows how the calculated decay constant varies with the scattering coefficient $\mu_{s}$ while the absorption coefficient is kept constant in the calculation. The decay constants are obtained in the depth range of two to three times the mean free path. The result shows that the calculated decay constant is greater than $\mu_{t}$ for a small $\mu_{s}$ but smaller than $\mu_{t}$ for a large $\mu_{s}$. This phenomenon cannot be explained by the single back-scattering theory and should be related with the multiple scattering property of OCT. When $\mu_{s}$ is small, the singly back-scattered light 


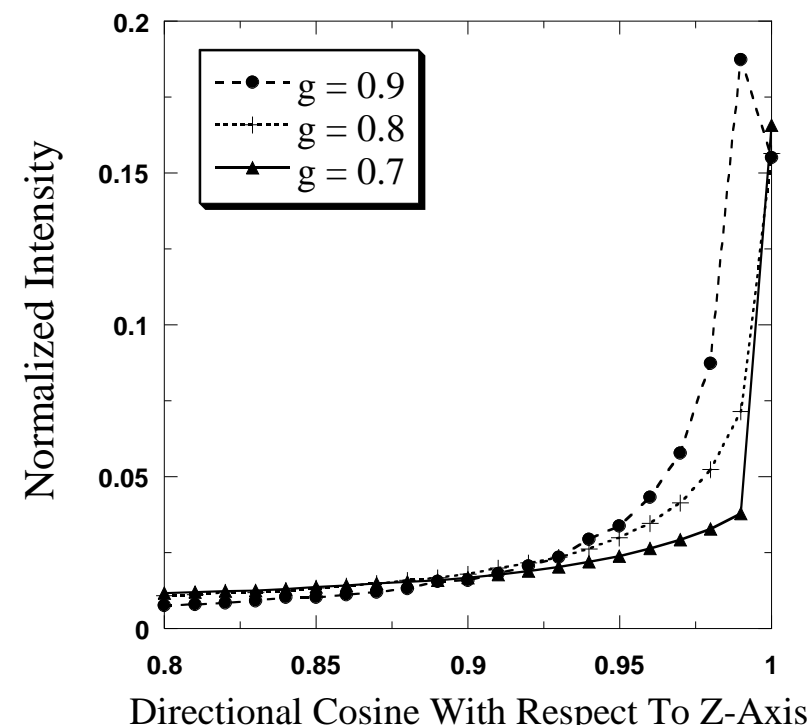

Figure 12. Angular distribution of the photons hitting the target layer (observed at the target), where the probing depth $=300 \mu \mathrm{m}$. The simulation parameters are the same as in figure 7 .

is dominant. For the same reason as discussed in section 3.2, the calculated decay constant should be greater than $\mu_{t}$. When $\mu_{s}$ is large, the multiply scattered light becomes dominant and leads to a smaller decay constant.

\section{Conclusion and discussion}

In this paper, the angle-biased Monte Carlo method has been used to study the optical coherence tomography signal in homogeneous turbid media. The OCT signal is divided into two categories: one includes the light back-scattered from the target layer (Class I) and the other includes the multiply scattered light from the background other than the target layer (Class II). The Class I signal is useful because it can furnish local optical information about the target layer. The Class II signal consists of multiply back-scattered photons which have not interacted with the target layer and should be avoided because it does not furnish information about the target layer. Simulation results (figure 5 and 6) show that these two classes of signals have very different spatial and angular distributions. The Class II signal has a wider spatial and angular distribution than the Class I signal and experiences more scattering events. Due to these differences, it is possible to reject the Class II photons by adding some spatial and angular limitations. However, the Class II signal will eventually become dominant at some imaging depths. The actual cross-over point is related to the efficiency of Class II signal rejection and on the imaging configuration.

The average number of scattering events increases linearly with the probing depth for both the Class I and Class II signals. However, the multiple scattering events associated with the Class I signal must be small-angle forward scattering, while the multiple scattering events associated with the Class II signal are large-angle back-scattering. This is because of the restriction of the optical path-length. In theory, the multiply scattered photons in the Class I signal also decrease the contrast of the OCT image because they experience interactions outside the target layer. 


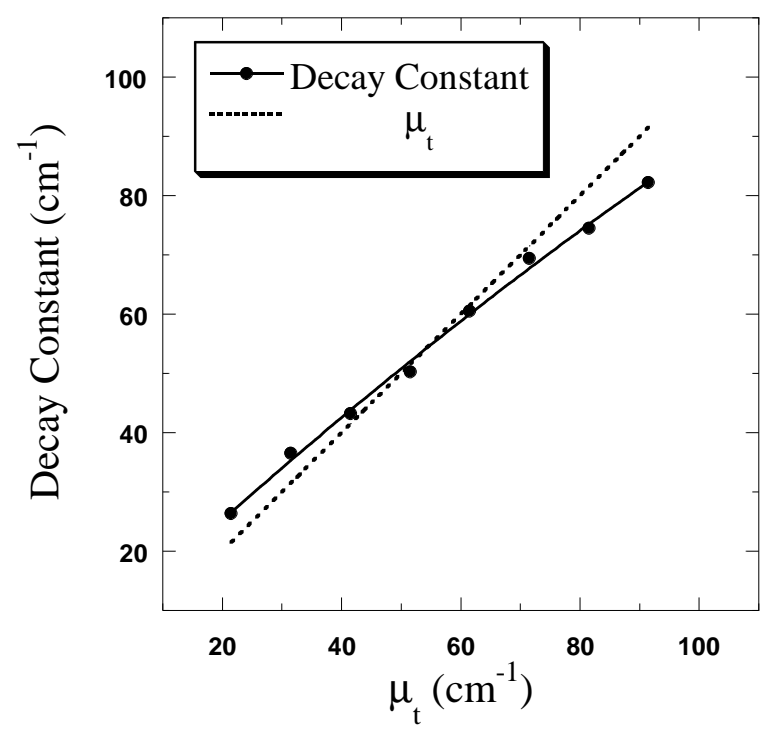

Figure 13. Decay constant of the Class I signal versus $\mu_{t}$, where $l_{c}=15 \mu \mathrm{m}, n=1.5$, $\mu_{a}=1.5 \mathrm{~cm}^{-1}, g=0.9, r_{d}=10 \mu \mathrm{m}$ and $\theta_{d}=5^{\circ}$.

The calculation shows that the decay constant of the Class I signal is close to the total attenuation coefficient $\mu_{t}\left(=\mu_{a}+\mu_{s}\right)$ and is affected by the coherence length of the OCT system as well as the optical properties $\left(\mu_{a}, \mu_{s}\right.$ and $\left.g\right)$. Because the Class I signal is dominant at small imaging depths, it is possible to measure the optical properties by measuring the attenuation of the OCT signal at small depths (Schmitt et al 1993). However, our simulation results show that the decay constant of the Class I signal is not exactly equal to the true attenuation coefficient (figure 13). The decay of the OCT signal is affected by the specific configuration that is used to couple the probing fibre and the sample. In addition, for measurements in heterogeneous media, the multiple scattering of Class I signal will also affect the accuracy of such measurements.

It must be pointed out that the current simulation did not consider the interference phenomena between the scattered light. The calculated signal is based on the ensemble averaged light intensity due to the inherent characteristic of Monte Carlo simulations. In reality, the spatial coherence of multiple scattered light will decrease (Schmitt and Knuttel 1997) and therefore yield a reduced heterodyne efficiency. From this point of view, our Monte Carlo simulation results can be considered as a worst case scenario. Nevertheless, this study directly reveals the nature of the competence of multiple scattering in an OCT signal and is helpful for better understanding and interpreting the OCT signals in turbid media. Our study will be extended to simulate heterogeneous scattering media and to incorpoarate the focusing scheme of an experimental set-up.

\section{Acknowledgments}

This project was sponsored in part by the National Institutes of Health grants R29 CA68562 and R01 CA71980 and by the National Science Foundation grant BES-9734491. 


\section{References}

Hendricks J S and Carter L L 1985 Anisotropic angle biasing of photons Nucl. Sci. Eng. 89 118-30

Huang D, Swanson E A, Lin C P, Schuman J S, Stinson W G, Chang W, Hee M R, Flotte T, Gregory K, Puliafito C A and Fujimoto J G 1991 Optical coherence tomography Science 254 1178-81

Pan Y T, Birngruber R, Rosperich J and Engelhardt R 1995 Low-coherence optical tomography in turbid tissue: theoretical analysis Appl. Opt. 34 6564-74

Puliafito C A, Hee M R, Lin C P, Reichel E, Schuman J S, Duker J S, Izatt J A, Swanson E A and Fujimoto J G 1995 Imaging of macular disease with optical coherence tomography Opthalmology 120 217-29

Schmitt J M and Ben-Letaief K 1996 Efficient Monte Carlo simulation of confocal microscopy in biological tissue J. Opt. Soc. Am. A $13952-61$

Schmitt J M and Knuttel A 1997 Model of optical coherence tomography of heterogeneous tissue J. Opt. Soc. Am. A 14 1231-42

Schmitt J M, Knuttel A and Bonner R F 1993 Measurement of optical properties of biological tissue by low coherence reflectometry Appl. Opt. 32 6032-42

Siegman A E 1966 The antenna properties of optical heterodyne receivers Appl. Opt. 5 1588-94

Smithies D, Lindmo T, Chen Z, Nelson J S and Milner T E 1998 Signal attenuation and localization in optical coherence tomography studied by Monte Carlo simulation Phys. Med. Biol. 43 3025-44

Sorin W V and Baney D M 1992 Measurement of Rayleigh backscattering at $1.55 \mu \mathrm{m}$ with $32 \mu \mathrm{m}$ spatial resolution IEEE Photonics Technol. Lett. 4 374-6

Wang L H, Jacques S J and Zheng L Q 1995 MCML-Monte Carlo modeling of photon transport in multi-layered tissue Comput. Methods Programs Biomed. 47 131-46

Yadlowsky M J, Schmitt J M and Bonner R F 1995 Multiple scattering in optical coherence microscopy Appl. Opt. 34 5699-707 